\title{
Relación cooperativa entre docentes y padres de estudiantes con discapacidad
}

Luz Mélida Montaño-Contreras luzmonco@unisabana.edu.co Universidad de La Sabana, Colombia ORCID: https://orcid.org/0000-0001-7430-9105

Johana Elizabeth Cerón-Bastidas

johanaceba@unisabana.edu.co

Universidad de La Sabana, Colombia ORCID: https://orcid.org/0000-0001-7335-4705

Mario Andrés Ernesto Martín-Padilla mario.martin1@unisabana.edu.co Universidad de La Sabana, Colombia ORCID: https://orcid.org/0000-0002-2497-768X

\section{Resumen}

El objetivo de la investigación fue identificar aspectos para la creación de una relación cooperativa entre maestros y familias con hijos en condición de discapacidad. Se realizó un estudio cualitativo, con un enfoque construccionista social. Los participantes fueron 32 padres de familia de estudiantes en condición de discapacidad y 16 maestros de dos instituciones educativas de Bogotá. Se utilizaron representaciones dibujadas y grupos de discusión. El procesamiento fue por codificación abierta y análisis narrativo. Como resultado se determinó una estructura con categorías y subcategorías para la relación cooperativa, la cual puede guiar un sistema interaccional conformado por padres y docentes.

\section{Palabras clave}

Relación padres-docente; educación integradora; incapacidad

\begin{abstract}
This research aims to identify the aspects involved in the creation of a cooperative relationship between teachers and families of students with any disability. It is a qualitative study conducted under a social constructivist approach. The participants were 32 parents of students with disabilities and 16 teachers from two educative institutions in Bogotá. Drawn representations and discussions groups were implemented. The processing consisted in open coding and narrative analysis. As a result, a structure with categories and subcategories was determined for the cooperative relationship, which would be useful as a guide for the interaction system between parents and teachers.
\end{abstract}

\section{Keywords}

Parents-teacher relationship; integrating education; disability

Para citar este artículo / To cite this article

Montaño-Contreras, L. M.; Cerón-Bastidas, J. E. \& Martín-Padilla, M. A. E. (2019). Relación cooperativa entre docentes y padres de estudiantes con discapacidad. magis, Revista Internacional de Investigación en Educación, 12 (24), 145-160. doi: 10.11144/Javeriana.m12-24.rcdp 


\section{Introducción}

Esta investigación tuvo el propósito de comprender los aspectos que inciden en el establecimiento de una relación cooperativa entre docentes y padres de familia, a fin de aportar elementos prácticos para facilitar la creación de contextos conversacionales durante el proceso de educación inclusiva para estudiantes en condición de discapacidad. Para que este proceso de la educación inclusiva se implemente exitosamente, es fundamental la participación efectiva de los padres de familia, asumiendo una responsabilidad compartida y complementaria con los educadores, para superar prejuicios, valorar la diversidad, y así alcanzar una mayor equidad en la educación (Calderón, 2012; Garnique-Castro \& Gutiérrez-Vidrio, 2012; Jiménez-León, 2008; Valdés \& Sánchez-Escobedo, 2016; Warren, Vialle \& Dixon, 2016). Para lograr este objetivo, se hace imprescindible fortalecer la comunicación, la información y la construcción de saberes entre el personal docente y los padres de familia, apoyándose mutuamente para afrontar los retos y vicisitudes de la educación para la promoción de la inclusión social (Turnbull, Turnbull \& Kyzar, 2009). Martha Blue-Banning, Jean Ann Summers, H. Corine Frankland, Louise Lord Nelson y Gwen Beegle (2004) plantean que para que este apoyo se dé es importante que en docentes y familias se promuevan actitudes como la calidad, la honestidad y el interés por querer conocer al otro. Igualmente, otro atributo crucial para la relación entre familia y escuela es la confianza mutua, la cual permite la expresión de necesidades en un diálogo abierto (Jiménez León, 2008; Peñafiel Martínez, 2012; Terrón, Alfonso \& Díez, 2003).

Desde esta perspectiva, para impulsar la inclusión educativa serían esenciales tanto un currículo flexible que se adapte a las características, los intereses y las necesidades del alumno, como promover un trabajo de cooperación entre el profesorado, el contexto terapéutico y el contexto familiar (Calderón, 2012; Duk \& Murillo, 2013). Como sostiene Marina García Carmona (2012):

Descripción del artículo |
Article description
Este artículo de investigación es derivado
de la investigación para la obtención del
título de Maestría en Psicología de la Sa-
lud y la Discapacidad "Comunicación entre
maestros y familias con hijos con discapaci-
dad en instituciones de educación inclusi-
va" y hace parte de la línea de Convivencia
y Contextos Socioculturales, del grupo de
investigación Procesos Psicológicos y Con-
texto Social.

Descripción del artículo |
Article description
Este artículo de investigación es derivado
de la investigación para la obtención del
título de Maestría en Psicología de la Sa-
lud y la Discapacidad "Comunicación entre
maestros y familias con hijos con discapaci-
dad en instituciones de educación inclusi-
va" y hace parte de la línea de Convivencia
y Contextos Socioculturales, del grupo de
investigación Procesos Psicológicos y Con-
texto Social.

Descripción del artículo |
Article description
Este artículo de investigación es derivado
de la investigación para la obtención del
título de Maestría en Psicología de la Sa-
lud y la Discapacidad "Comunicación entre
maestros y familias con hijos con discapaci-
dad en instituciones de educación inclusi-
va" y hace parte de la línea de Convivencia
y Contextos Socioculturales, del grupo de
investigación Procesos Psicológicos y Con-
texto Social.

Descripción del artículo |
Article description
Este artículo de investigación es derivado
de la investigación para la obtención del
título de Maestría en Psicología de la Sa-
lud y la Discapacidad "Comunicación entre
maestros y familias con hijos con discapaci-
dad en instituciones de educación inclusi-
va" y hace parte de la línea de Convivencia
y Contextos Socioculturales, del grupo de
investigación Procesos Psicológicos y Con-
texto Social.

Descripción del artículo |
Article description
Este artículo de investigación es derivado
de la investigación para la obtención del
título de Maestría en Psicología de la Sa-
lud y la Discapacidad "Comunicación entre
maestros y familias con hijos con discapaci-
dad en instituciones de educación inclusi-
va" y hace parte de la línea de Convivencia
y Contextos Socioculturales, del grupo de
investigación Procesos Psicológicos y Con-
texto Social.

Descripción del artículo |
Article description
Este artículo de investigación es derivado
de la investigación para la obtención del
título de Maestría en Psicología de la Sa-
lud y la Discapacidad "Comunicación entre
maestros y familias con hijos con discapaci-
dad en instituciones de educación inclusi-
va" y hace parte de la línea de Convivencia
y Contextos Socioculturales, del grupo de
investigación Procesos Psicológicos y Con-
texto Social.

Descripción del artículo |
Article description
Este artículo de investigación es derivado
de la investigación para la obtención del
título de Maestría en Psicología de la Sa-
lud y la Discapacidad "Comunicación entre
maestros y familias con hijos con discapaci-
dad en instituciones de educación inclusi-
va" y hace parte de la línea de Convivencia
y Contextos Socioculturales, del grupo de
investigación Procesos Psicológicos y Con-
texto Social.

Descripción del artículo |
Article description
Este artículo de investigación es derivado
de la investigación para la obtención del
título de Maestría en Psicología de la Sa-
lud y la Discapacidad "Comunicación entre
maestros y familias con hijos con discapaci-
dad en instituciones de educación inclusi-
va" y hace parte de la línea de Convivencia
y Contextos Socioculturales, del grupo de
investigación Procesos Psicológicos y Con-
texto Social.

Descripción del artículo |
Article description
Este artículo de investigación es derivado
de la investigación para la obtención del
título de Maestría en Psicología de la Sa-
lud y la Discapacidad "Comunicación entre
maestros y familias con hijos con discapaci-
dad en instituciones de educación inclusi-
va" y hace parte de la línea de Convivencia
y Contextos Socioculturales, del grupo de
investigación Procesos Psicológicos y Con-
texto Social.

El sentido de comunidad que aflora al trabajar cooperativamente ofrece una riqueza especial al proceso de enseñanza-aprendizaje. De este modo se evitarán las incongruencias de la educación, ya que el alumno obtendrá respuestas educativas semejantes tanto en casa como en el centro escolar (p. 66).

\section{Antecedentes en la relación padres-educadores para la inclusión}

Los estudios sobre los efectos de la cooperación entre las familias y el personal docente muestran que esta ha favorecido al estudiante en condición de discapacidad en aspectos como el apoyo familiar a la realización de las tareas escolares, y ha desarrollado en los padres el interés, la disposición y la comunicación constante con los docentes, lo cual ha repercutido en la autoestima y seguridad de los alumnos (Fontana, Alvarado, Angulo, Marín \& Quirós, 2009). Sin embargo, para que esta cooperación sea eficaz se requiere del liderazgo del docente como agente mediador, es decir, que tome la misión de promover una toma de decisiones en que todas las distintas voces de la familia y la comunidad se vean reflejadas, y haya una comunicación abierta y transparente de las acciones y determinaciones en el proceso educativo (Sales-Ciges, Ferrández-Berrueco \& Molinet-García, 2012).

En este sentido, en distintos países, investigaciones sobre la participación de los padres en el proceso educativo en la escuela indican que en 
varios casos hay dificultades tanto en padres de familia como en educadores para trabajar de forma cooperativa. En un estudio en Suecia con padres en la escuela primaria, por ejemplo, se vio mayor poder de los maestros para incidir en cómo los padres educan a sus hijos que en los padres para influir en la educación que se da en la escuela (Widding \& Berge, 2014). De otra parte, hay desconfianza en los padres sobre la competencia del sistema educativo para responder a las necesidades de todos los estudiantes, como se encontró en un estudio sobre las actitudes de padres con hijos sin discapacidad frente a la educación inclusiva (Dimitrova-Radojichich \& Chichevska-Jovanova, 2014). Así mismo, un estudio en México sobre la perspectiva de los docentes (Valdés \& Sánchez-Escobedo, 2016) muestra que para ellos hay poca participación de los padres de familia en la escuela por su poca disponibilidad de tiempo, poco interés y excesivas demandas laborales a que son sometidos. Hay poco trabajo cooperativo; los docentes están poco dispuestos a ver a los padres de familia como pares educadores que puedan aportar desde sus experiencias con sus hijos, ni los han involucrado en la toma de decisiones, lo que hace que no se promuevan otras formas de acercamiento con ellos (Sukys, Dumciene \& Lapeniene, 2015; Valdés \& Sánchez-Escobedo, 2016).

Otra limitante reportada en la educación es que muchas veces los docentes no dan participación a los niños con barreras para el aprendizaje, y no interactúan con ellos, debido a sus propias representaciones sobre discapacidad y sus bajas expectativas sobre las capacidades de estos niños (Damm, 2010; Valdés \& Sánchez-Escobedo, 2016). En este sentido, para que los docentes puedan redefinir sus creencias, es indispensable que el profesorado tenga apertura al cambio, investigue y se actualice en el tema de la inclusión educativa (Fernández-Batanero, 2013).

De otra parte, al estudiar procesos de inclusión educativa en Perú, Meybol Calderón (2012) encuentra que los padres de familia muchas veces son los que dificultan la interacción de sus hijos con sus pares al asumir actitudes de sobreprotección, en el afán de protegerlos de burlas o de actitudes de rechazo.

\section{Construcción social de las relaciones cooperativas}

La propuesta de estudiar los aspectos que facilitan o dificultan una relación cooperativa entre docentes y padres de familia en la inclusión educativa apunta a generar un proceso conversacional desde los niveles epistemológico, metodológico y pragmático (Valles, 1999). Desde el punto de vista epistemológico, para la construcción del conocimiento, el trabajo toma como referente el construccionismo social y el lenguaje de interacción social, como proceso de comprensión de la realidad (Gergen, 1996). Desde esta postura, "es el lenguaje el que posibilita la coconstrucción de acciones conjuntas entre quienes comparten contextos específicos y estas acciones tienen significados para quienes intervienen en ellas y las comprenden" (Agudelo-Bedoya \& Estrada, 2013, p. 365).

En cuanto a lo metodológico, se muestran la aplicación de la educación inclusiva desde el modelo ecológico y las distinciones entre los subsistemas involucrados, para la construcción de los principios operadores de un sistema funcional de significados y coordinaciones de acciones, que sería compuesto por dos sistemas primarios de influencia inmediata para los estudiantes, como son familia y escuela, que, al interactuar, formarían un sistema de orden superior, o mesosistema (Bronfenbrenner, 2002). En lo que se refiere a lo pragmático, se busca develar y redefinir los componentes de la relación cooperativa en el mesosistema conformado por 
familia-escuela, como contexto interaccional donde se sitúa el proceso investigado. En este orden, se analizan las narrativas dominantes, es decir, aquellos relatos sobre la experiencia que se enquistan como si fueran irreductibles, con lo cual se predeterminan las interacciones y se niegan posibles alternativas en las relaciones (White \& Epston, 1993). En este sentido, autores como Martha Blue-Banning, Jean Ann Summers, H. Corine Frankland, Louise Lord Nelson y Gwen Beegle (2004), y sobre este trabajo, Ann P. Turnbull, H. Rutherford Turnbull y Kathleen Kyzar (2009) han resumido los atributos que pueden caracterizar una relación cooperativa: comunicación, que debe ser de doble vía, frecuente, abierta, y honesta; compromiso, tanto de los docentes con su disponibilidad y dedicación profesional, como de los padres con las tareas educativas; equidad, o sea, reciprocidad, que se ve "en expresar opiniones y ayudar a las familias a adquirir habilidades que les permitan participar plenamente en la toma de decisiones" (Blue-Banning, Summers, Frankland, Nelson \& Beegle, 2004, p. 177), competencia profesional, es decir, habilidades del docente para lograr adaptar sus instrucciones a las necesidades del niño y asegurar su progreso, respeto, es decir, tratarse con consideración, dignidad y sin prejuicios, y confianza, que implicaría mantener la confidencialidad, cumplir lo que se dice, hacer juicios bien fundados y salvaguardar la seguridad de los participantes para no ser dañados en su dignidad. Estos elementos son claves en la construcción de nuevas posibilidades en las narrativas sobre las relaciones para el manejo de una comunicación abierta en un ambiente de confianza, donde se puedan exponer las posturas y puntos de vista, así como las dudas e interrogantes, lo que puede facilitar en padres y educadores la creación de nuevas comprensiones y posibilidades de apoyo mutuo (Sales-Ciges, Ferrández-Berrueco \& Molinet-García, 2012).

Así, el objetivo de este estudio fue identificar aspectos para la creación de una relación cooperativa entre maestros y familias con hijos en condición de discapacidad, a partir de las percepciones de padres y educadores sobre la relación que se ha dado en el contexto escolar, en instituciones que están llevando a cabo procesos de educación inclusiva.

\section{Enfoque metodológico y diseño de investigación}

Se realizó un estudio descriptivo cualitativo, desde un enfoque social interpretativo (Corbetta, 2007). Los participantes, convocados de manera intencional, fueron 32 padres de familia de estudiantes en condición de discapacidad (16 padres de familia por colegio) y 16 maestros (8 maestros por colegio), seleccionados en dos instituciones educativas de Bogotá, los cuales debían cumplir el requisito de tener en sus aulas estudiantes en condición de discapacidad. No se tuvo en cuenta como criterio de inclusión o de análisis el tipo de discapacidad de los jóvenes, dado que el foco en este trabajo fue el definir parámetros generales para la construcción de la relación cooperativa entre padres y docentes para la inclusión. Los directivos de los colegios y los participantes (maestros y familias) firmaron un consentimiento informado.

Se llevaron a cabo sesiones de dibujo y grupos de discusión, en los que se obtuvieron textos escritos y narrados en las técnicas de recogida de información (Caillé, 1992; White \& Epston, 1993). Las narraciones de las experiencias se toman como unidades de significado, para conversar e interpretar la experiencia vivida (Epston, White \& Murray, 1996). Como estrategia de validación y credibilidad, así como de contrastación, se utilizó la triangulación de participantes tomando dos muestras distintas (maestros y familias en condición de discapacidad) y de técnicas (representaciones 
dibujadas y grupo de discusión) (Denzin \& Lincoln, 2000; Okuda \& Gómez-Restrepo, 2005), para la validación interna de la investigación y poder enriquecer los resultados. Para la categorización se utilizaron de forma deductiva los atributos de una relación cooperativa: comunicación, compromiso, equidad, competencia profesional, respeto y confianza (BlueBanning, Summers, Frankland, Nelson \& Beegle, 2004; Turnbull, Turnbull \& Kyzar, 2009).

\section{Instrumentos}

\section{Representaciones en dibujo}

El propósito de esta técnica es utilizar un canal no verbal para expresar emociones y condensar de forma simbólica, a través de una expresión visual las vivencias, mediante "una producción dialógica y situada del conocimiento que tiene entre sus recursos la imagen audiovisual, las memorias recreadas en los recorridos, las historias y relatos de vida, las fotografías y los archivos gráficos, el dibujo y la expresión creativa" (Montoya-Arango, García-Sánchez \& Ospina-Mesa, 2013, p. 192). Esta técnica puede favorecer la expresión y resignificación de las narrativas, al disminuir sesgos y barreras racionales que pudieran presentarse en una conversación verbal directa; según Philippe Caillé (1992), el simbolismo de la creación artística desafía los estereotipos. Cada dibujo fue acompañado de un breve texto que describe o explica la situación; esta combinación de texto y dibujo como formas narrativas permite, retomando a autores como Michael White y David Epston (1993), construir una "unidad de idea" (p. 51), que puede sintetizar la información e incrementar su contenido, pues permite al dibujante procesar la experiencia que quiere plasmar, por medio de una frase elaborada a partir de su producción ilustrada.

Para el procedimiento, se dieron materiales a padres y docentes, con la consigna de dibujar una escena que ilustrara la relación padres-docentes en la escuela; en los dibujos y su posterior descripción oral, se busca develar tres elementos constitutivos del análisis: a) las situaciones problematizadas, b) las partes implicadas (los actores) y c) las percepciones positivas o negativas. Con estos elementos, se planteó a los participantes conversar sobre las escenas dibujadas con una técnica complementaria, el grupo de discusión.

\section{Grupo de discusión}

El grupo de discusión es una técnica cualitativa que busca verificar las ideas pertinentes o válidas mediante un espacio grupal, en que se hagan explícitos los puntos de vista y puedan debatirse (Canales \& Peinado, 1995). El propósito de usar esta técnica es trasladar las expresiones simbólicas de los dibujos al nivel verbal, para pasar de lo individual a lo colectivo y para la construcción de relatos enriquecidos por el proceso conversacional; así, por medio del grupo de discusión se canalizan los consensos y diferencias sobre los aspectos plasmados en las representaciones. Como dinámica, se buscó que cada participante relatará de manera abierta lo que describía su representación en dibujo. La conversación fue orientada a las percepciones sobre las descripciones de las escenas dibujadas; los investigadores animaron la expresión de consensos y disensos sobre los siguientes tópicos, según el rol:

- Docentes: tipo de comunicación para mantenerse en contacto con las familias, información que brindan a los padres, si tienen escucha activa, a quien informan cuando tienen dificultades, si 
conocen las habilidades de los padres, si explican el proceso educativo, si han hecho encuentros con los padres para hablar de necesidades e intereses o intercambiar experiencias, si han usado estrategias para que los padres participen en las decisiones, y actitudes que perciben en ellos frente al proceso de inclusión.

- Padres: conocimiento de las estrategias del colegio para la inclusión educativa y la participación de los padres, interés por participar en estos procesos, por qué medio se enteran del proceso educativo de su hijo, participación en las decisiones sobre este proceso, si ha habido cooperación padres-docentes, actitudes de los docentes frente al proceso educativo y la inclusión, conocimiento de los derechos de su hijo e interés en cooperar con el docente.

Procedimiento

El procedimiento se construyó en tres fases:

Fase 1. Sesión de dibujos

Se trabajó con dos grupos, uno por cada institución educativa. En cada grupo se convocó a 16 padres de familia y 8 docentes en cada sesión; a cada persona se le proporcionaron materiales (papel, marcadores) para que elaborara un dibujo con la consigna de plasmar su percepción de la relación entre padres y docentes. Posteriormente, cada persona explicó su dibujo.

\section{Fase 2. Grupos de discusión}

En una segunda sesión, se convocó a 16 padres y los 8 maestros que participaron en cada uno de los dos grupos.

\section{Fase 3. Análisis interpretativo de textos escritos y hablados}

Se tomaron los textos escritos en los dibujos, así como las explicaciones de los mismos por parte de los participantes; igualmente, se tomaron las opiniones del grupo de discusión, para la interpretación de los contenidos y la definición de los componentes de la relación docentes-padres de familia. Posteriormente, se codificaron los componentes de la relación cooperativa docentes-padres de familia en matrices, para elaborar las categorías y subcategorías y conceptualizarlas mediante un proceso de teorización (Strauss \& Corbin, 2002).

\section{Relación cooperativa docentes-padres: categorización de componentes}

Una vez se interpretaron los resultados, se desarrollaron las categorías y se codificaron los contenidos de forma abierta, en un proceso deductivo-inductivo, para la construcción de categorías y subcategorías que se identificaron en la relación cooperativa docentes-familia. Estas categorías son: habilidades sociales comunicativas, no solo las macrolingüísticas (leer, escuchar, hablar, escribir), sino también las destrezas y recursos personales para la socialización e intercambio (Cevallos-Mieles, 2016); aspectos actitudinales internos y externos, es decir, la predisposición hacia otros miembros, hacia el trabajo colaborativo, y hacia la inclusión (Angenscheidt-Bidegain \& Navarrete-Antola, 2017); y cumplimiento de roles, que implica el compromiso e involucramiento de padres y la capacidad de los docentes para cumplir sus responsabilidades familiares y profesionales (Angenscheidt-Bidegain \& Navarrete-Antola, 2017; González-Rojas \& Triana-Fierro, 2018). 
Tabla 1

Categorías y componentes de la interacción familia-escuela en la educación inclusiva

\begin{tabular}{|c|c|c|}
\hline Categorías & Subcategorías & Definición conceptual \\
\hline \multirow{4}{*}{$\begin{array}{l}\text { Categoría I: } \\
\text { habilidades } \\
\text { sociales } \\
\text { comunicativas }\end{array}$} & $\begin{array}{l}1.1 \text { Intercambio } \\
\text { dialógico }\end{array}$ & $\begin{array}{l}\text { El acto comunicativo donde todos aprenden sin tener en cuenta las jerarquías } \\
\text { de conocimiento, en que los interlocutores buscan una comprensión } \\
\text { significativa y concientizadora de los problemas (Freire, 1998). }\end{array}$ \\
\hline & 1.2 Empatía & $\begin{array}{l}\text { En el proceso de empatía en la escuela, se les debe dar importancia a la } \\
\text { capacidad y a la destreza para identificar y comprender los sentimientos } \\
\text { de los demás. }\end{array}$ \\
\hline & 1.3 Asertividad & $\begin{array}{l}\text { La formación asertiva está diseñada para identificar y reemplazar mensajes de } \\
\text { sumisión y de abnegación por afirmaciones que reflejan con más precisión lo } \\
\text { que el individuo siente, necesita y desea por sí mismo. La formación asertiva } \\
\text { alienta a las personas a aprender a decir no (Trevithick, 2006). }\end{array}$ \\
\hline & 1.4 Cooperación & $\begin{array}{l}\text { Involucrar a las familias en la escuela fortalecerá el compromiso, el } \\
\text { empoderamiento y la responsabilidad, para construir soluciones no a través de } \\
\text { las dificultades de las familias con hijos en condición de discapacidad, sino a } \\
\text { través de los facilitadores como la experiencia de conocer y saber qué necesita y } \\
\text { qué le interesa a su hijo. }\end{array}$ \\
\hline \multirow[t]{3}{*}{$\begin{array}{l}\text { Categoría } \\
\text { II: aspectos } \\
\text { actitudinales } \\
\text { internos y externos }\end{array}$} & $\begin{array}{l}2.1 \text { Manejo de } \\
\text { la frustración }\end{array}$ & $\begin{array}{l}\text { La frustración se presenta de dos maneras: (a) En la familia. Según Ann Turnbull } \\
\text { y H. Rutherford Turnbull (1990), cada proceso que atraviesa la familia de la } \\
\text { persona en condición de discapacidad crea una coraza de asimilación, en la } \\
\text { cual cada miembro sufre la frustración e intenta entender la discapacidad de la } \\
\text { mejor manera posible; se rompe el imaginario de ese hijo tan anhelado. (b) En } \\
\text { los maestros: también pasan por un proceso de frustración y aceptación a causa } \\
\text { de tener en su aula un alumno en condición de discapacidad; su actitud positiva } \\
\text { o negativa va a reflejar dicha aceptación (Damm, 2010). }\end{array}$ \\
\hline & 2.2 Confianza & $\begin{array}{l}\text { Para Luis Alfonso Morey (2009), "es fácil perder la confianza en alguien cuando } \\
\text { esa persona con quien tratamos no actúa con justicia y transparencia" (p. 36). } \\
\text { Por esto, es importante que tanto las familias como los maestros se arriesguen } \\
\text { a confiar uno en el otro, en pro del alcance de sus metas u objetivos como } \\
\text { herramienta de éxito. }\end{array}$ \\
\hline & 2.3 Respeto & $\begin{array}{l}\text { "En el respeto, conformamos nuestro criterio al valor fundamental de lo } \\
\text { existente; lo reconocemos, damos en cierto modo a lo existente la oportunidad } \\
\text { de desplegarse, de que nos hable, de que fecunde nuestro espíritu" (Von } \\
\text { Hildebrand, 2004, p. 222). En la relación familia y escuela es el primer paso de } \\
\text { cortesía para consolidar la comunicación. }\end{array}$ \\
\hline \multirow[t]{2}{*}{$\begin{array}{l}\text { Categoría III: } \\
\text { cumplimiento } \\
\text { de roles }\end{array}$} & 3.1 Compromiso & $\begin{array}{l}\text { La familia y la escuela deben comprometerse respecto a brindar una atención } \\
\text { responsable y de calidad en los ámbitos educativo, médico, recreativo, } \\
\text { vocacional, entre otros. }\end{array}$ \\
\hline & $\begin{array}{l}\text { 3.2 Competencia } \\
\text { Profesional }\end{array}$ & $\begin{array}{l}\text { Un buen profesor ha de ser, no solo el que es experto en su } \\
\text { materia, sino también el que comprende su quehacer desde el compromiso } \\
\text { y ayuda hacia el otro, desde la responsabilidad de su labor. Maestros } \\
\text { técnicamente capaces y éticamente exigentes (Martínez Navarro, 2010). }\end{array}$ \\
\hline
\end{tabular}

Fuente: elaboración propia

\section{Resultados por categoría}

\section{Categoría I: habilidades sociales comunicativas}

Al interpretar lo dicho por familiares y maestros, se puede deducir que no ha habido un intercambio dialógico eficaz; las conversaciones que sostienen los docentes con los padres son esporádicas y centradas en los aspectos académicos y disciplinarios, más que en el proceso de adaptación o en la toma de decisiones sobre el manejo de la discapacidad, como se ve en el siguiente enunciado de un maestro: 
Los padres no escuchan, ni se interesan por aprender; se debe conversar más para llegar a acuerdos (texto en la representación dibujada elaborada por un maestro').

Esta falta de comunicación incide en la empatía, pues los maestros y las familias no conocen o se interesan por las dificultades particulares de cada docente o cada familia:

Los maestros no muestran interés por conocer los problemas y preocupaciones que pasan en las familias de sus alumnos (texto en la representación dibujada elaborada por la familia).

Así se pudo establecer que los maestros y las familias ven con prevención la actitud de la contraparte; esto les impide conversar asertivamente y desarrollar una relación de cooperación:

Padres y maestros siempre debemos formar un equipo unido; sin embargo, en más de una ocasión por malos entendidos vamos por caminos distintos, sin entender que esto solo perjudica a nuestros alumnos (Maestro, grupo de discusión).

Resultan sorprendentes la distancia, el recelo y enfrentamiento constante que hemos tenido con algunas instituciones o maestros de mi hijo, si nosotros como familia y los maestros buscamos el mismo fin, que es la mejor educación de los niños y eso es lo más importante (Padre, grupo de discusión).

\section{Categoría II: aspectos actitudinales internos y externos}

Se pudo evidenciar que tanto las familias con hijos en condición de discapacidad como los maestros atraviesan momentos de frustración relacionados con la dificultad personal de afrontar la discapacidad del niño. Cuando se habla de frustración entre los maestros, se expone que ellos inician con un proceso de no aceptación de tal responsabilidad, que fortalecen al afirmar que no cuentan con las competencias para atender estudiantes en condición de discapacidad, y culpabilizan de esto a la institución educativa; incluso hubo maestros que manifestaron haber tenido situaciones graves de salud, debido al estrés, por lo que han buscado que el estudiante sea atendido en otro lugar.

En cuanto a la frustración familiar, cuando al hogar llega una persona en condición de discapacidad, frecuentemente hay culpa, acusaciones mutuas y sobreprotección de los hijos en condición de discapacidad, por lo cual los padres subvaloran las posibilidades del niño:

Soy consciente que en muchas ocasiones he negado la posibilidad de avance de aprendizaje que puede llegar a tener mi nieto, demeritándolo, afirmando que él no puede, es incapaz, es minusválido, no sabe (Padre, grupo de discusión).

Así mismo, docentes y padres han tenido dificultades para promover un clima de confianza y respeto, lo que a su vez no ha favorecido que familias y educadores se apoyen frente a las reacciones emocionales negativas que se pueden experimentar al asumir la tarea de la educación inclusiva. Los padres consideran que, a veces, los maestros no son cuidadosos con la

1 Magis no edita ni corrige las entrevistas realizadas en las investigaciones; por eso, las citas contienen marcas del lenguaje oral, muletillas, errores... 
información y no actúan coherentemente en el aula, y los maestros perciben que los padres son recelosos y los vigilan:

Cuando nuestros niños ingresan, no les tienen en cuenta sus habilidades; son vistos como un problema, y están enfocados en el diagnóstico (Padre, escrito en la representación en dibujo).

Las familias están muy lastimadas y predispuestas a romper cualquier vínculo de cordialidad; en la jornada de los descansos, los familiares se ubican en la reja fuera del colegio para vigilar a sus hijos y a mí (Maestro, grupo de discusión).

Para los maestros, las familias no forman a sus hijos para el respeto:

La familia debe educar respecto a valores indispensables para la sana convivencia y armonía, dejando de lado aspectos culturales que afectan los procesos comunicativos tales como las palabras soeces o querer solucionar todo agresivamente (Maestro, grupo de discusión).

\section{Categoría III: cumplimiento de roles}

En cuanto a la percepción sobre el cumplimiento de roles, se hicieron cuestionamientos sobre el compromiso de padres y educadores, y sobre la forma en que se están asumiendo las responsabilidades. Referente a los planes caseros y el cumplimiento de tareas en casa, los docentes piensan que los padres - por su carga laboral y familiar o por delegar su responsabilidad - no quieren actividades extra:

La familia olvida sus responsabilidades y deberes para con su hijo (Maestro, escrito en la representación en dibujo).

No hay una intención por parte del familiar por dar a conocer la realidad del estado de salud de su hijo... la familia debe estar más comprometida con cada uno de los aspectos que conciernen a su hijo, especialmente aspectos de la salud. La familia no cumple en las estrategias que se les brinda como planes caseros, estrategias pedagógicas entre otros, que son fundamentales para el proceso de educación de su hijo (Maestro, grupo de discusión).

Así mismo, para las familias, en cuanto al compromiso, los docentes a veces presentan dificultades al asumir el proceso de inclusión, muchas veces por verlo como una carga extra, por miedo hacia lo desconocido o por timidez:

Cuando mi hijo se ausenta, no hay un interés del porqué se ausentó, para la maestra es mejor que no vaya a estudiar porque es más trabajo para ella (Padre, grupo de discusión).

Me doy cuenta que no se está haciendo una evaluación académica inadecuada $[s i c]$, porque mi hijo lleva tres años repitiendo tercero. La promoción no se realiza de acuerdo a las habilidades de cada estudiante (Padre, grupo de discusión).

En la escuela solo recibimos citaciones de aspectos negativos de nuestros hijos, nunca somos citados para felicitarnos por las habilidades o logros pequeños (Padre de familia, representación en dibujo).

Los maestros, sin embargo, reconocen que no se han promovido espacios de encuentro donde tanto maestros como familias expresen más abiertamente sus habilidades y cualidades, actitud que puede coincidir con 
lo encontrado por Ángel Alberto Valdés y Pedro Antonio Sánchez Escobedo (2016), en cuanto a la dificultad de los docentes para concebir que los padres de familia puedan ser pares educadores.

De otra parte, con respecto a la competencia profesional:

Me siento en desventaja con mis demás compañeros, porque yo debo atender a este estudiante, y yo no tengo la competencia para educarlo; además, es frustrante saber que el estudiante no avanza por más que te esfuerzas (Maestro, representación en dibujo).

Esto confirma lo hallado en otros estudios (Angenscheidt-Bidegain \& Navarrete-Antola, 2017; González-Rojas \& Triana-Fierro, 2018): la actitud negativa de los docentes ante la inclusión puede deberse en parte a que no están suficientemente capacitados para dar respuesta a este proceso, por lo que, según los autores consultados, se hace necesario el trabajo en su práctica pedagógica, en la búsqueda de recursos y en el aprendizaje de estrategias didácticas de atención a la diversidad.

\section{Expectativas de cambio: relaciones cooperativas padres-docentes}

Como relatos alternativos al discurso dominante de la problematización, las familias manifestaron que sienten la necesidad de romper sus miedos y atreverse a ser más partícipes de la escuela, para brindar información clara respecto al estado de salud de su hijo. Esta proposición coincide con el planteamiento de Jordi Garreta i Bochaca (2007), acerca de la importancia de que los padres se impliquen en el proceso educativo de la escuela en su justa medida, mediante una buena comunicación y un buen conocimiento del medio en el que viven sus hijos.

Así mismo, los maestros reportaron que las familias mostraron interés en dar a conocer a sus hijos y en cumplir con tratamientos terapéuticos ausentes en los estudiantes. Tanto los maestros como las familias identificaron la importancia de establecer canales comunicativos en los que se dé un diálogo horizontal que desplace las jerarquías de poder y rompa el imaginario social respecto a quién tiene el conocimiento, lo que coincide con el concepto de conversación dialógica (Freire, 1998); así se pueden construir aprendizajes conjuntos que darán como resultado una mejor atención educativa y social para las personas en condición de discapacidad, como se ilustra en las siguientes afirmaciones:

El conocimiento del padre de familia es un tesoro para mí porque es él el que vive veinticuatro horas con el estudiante en condición de discapacidad, él conoce sus gustos, todo su ser. En mi caso, particularmente, fue un padre de familia el que me enseñó a manejar la convulsión de su hijo (Maestro, grupo de discusión).

Como padre de familia considero indispensable las orientaciones de la maestra en medio de este proceso tan duro, fue la maestra de mi hijo quien me mostró que había posibilidades para él (Padre de familia, grupo de discusión).

Se muestra la necesidad de padres y maestros de acercarse y comprenderse mutuamente, debido a su estado emocional y a las características de su personalidad (empatía). En este sentido, para los participantes es importante estar en disposición de poder expresar todas las dudas sin temor (confianza), y no rechazar las emociones que el otro manifiesta, lo 
que confirma lo hallado en otros estudios (Jiménez-León, 2008; PeñafielMartínez, 2012; Terrón, Alfonso \& Díez, 2003). Es decir, escucharse de una forma abierta sin ningún tipo de prejuicio y sin actitudes de rechazo:

Nuestros alumnos de inclusión educativa llegarán tan alto como nosotros se lo propongamos, ya que sus familias son capaces de sacrificarse, por lo que vale la pena y están empoderadas del proceso que tenemos que hacer en la institución y en la casa (Maestro, grupo de discusión).

Reconocemos que la labor de educar es un duro trabajo y más con nuestros niños que son especiales, agradezco a los maestros por su servicio y paciencia con mi hijo para que él aprenda cosas nuevas (Padre de familia, grupo de discusión).

De esta forma, padres y maestros reconocieron la importancia de hacer un trabajo conjunto. Sin embargo, para ellos es importante discutir los puntos de vistas diferentes en relación con determinados aspectos de la participación en educación inclusiva. Tener compromiso en los maestros para involucrar a las familias y enseñarles a convertirse en tutores de sus hijos en casa, y así apoyar las actividades y tareas en casa, e informarles con más regularidad sobre el desarrollo del niño, etc. Así mismo, muchos padres estiman y aceptan que algunas funciones ejercidas por la escuela les corresponden a ellos y muestran cada vez mayor interés en participar en tareas de gestión en la escuela:

Las ideas de la familia son fundamentales para la escuela, ellos son pieza importante para el proceso formativo; en ocasiones, tomamos decisiones sin preguntarles y eso les molesta; nosotros queremos que ellos conozcan las buenas intenciones que tenemos con sus hijos (Maestro, grupo de discusión).

Yo antes pensaba que era la única persona a la cual le interesaba mi hija, es importante saber que otras personas quieren ayudarle, ahora dejo a mi hija con más tranquilidad en el colegio y yo puedo dedicarme a otras actividades (Padre, grupo de discusión).

Esto coincide con la afirmación de Marta Sandoval-Mena, Cecilia Simón-Rueda y Gerardo Echeita-Sarrionandia (2012), en el sentido de que, para mejorar el proceso de educación inclusiva, es importante dirigirse mutuamente con una actitud de respeto y aceptación, para valorarse desde sus diferencias de rol:

El padre de familia reconoce mis esfuerzos, e inclusive me instruye en las cosas que ve que no estoy haciendo bien; yo recibo sus recomendaciones con agrado (Maestro, grupo de discusión).

Se destacó también en los participantes la importancia del reconocimiento, la expresión de la emoción, la identificación de lo que es necesario reparar en sí mismos y en la relación con los otros, para la coconstrucción de alternativas en las relaciones familia-escuela, en busca de relaciones menos jerárquicas y más igualitarias y participativas.

Igualmente, los participantes discutieron que, ante las frustraciones, es necesario que los maestros orienten a las familias en potenciar el desarrollo de la autoestima, la afectividad emocional respecto a la exploración de sentimientos y emociones, y la promoción de la integración social con otras familias, para compartir experiencias e identificar emociones, para que de esta manera se brinde un espacio para compartir y dilucidar sentimientos y emociones (manejo de la frustración). Por esto, como un logro 
del procedimiento investigativo, se encontró que los participantes tuvieron la posibilidad de analizar estos problemas, para fomentar relaciones de cercanía e identidad entre padres y maestros, para recrear realidades posibles mediante la construcción conjunta de significados (Berger \& Luckmann, 2001).

\section{Consideraciones finales}

Este estudio tuvo el propósito de aproximarse a las percepciones de los docentes y padres de familia con hijos en condición de discapacidad sobre su interacción frente a la inclusión, tanto para detectar las barreras percibidas, como para facilitar el diseño de estrategias de mejoramiento partiendo de la caracterización de componentes claves en esta interacción y de las propuestas de los participantes. En principio, se evidenció que el proceso de implementar una educación inclusiva ha sido una labor complicada, como lo han visto otros autores (Damm, 2010; Valdés \& SánchezEscobedo, 2016). Padres y maestros perciben que el proceso de inclusión escolar ha tenido para ellos dificultades y vicisitudes, relacionadas muchas veces con el contexto comunicacional creado por familias e instituciones, donde no ha habido espacios para el acercamiento. Igualmente, hay obstáculos para lograr un acercamiento entre las partes, debido a la diversidad de intereses, temores y expectativas, o a las barreras percibidas. En cuanto a estas barreras, tomando los aspectos para una relación cooperativa definidos por Blue-Banning, Summers, Frankland, Nelson y Beegle (2004), en la comunicación, ambas partes consideran que los canales establecidos entre la familia y la escuela no son efectivos por la poca frecuencia, y la prevención para hablar abiertamente de sus necesidades e inquietudes. Sobre el compromiso, las familias expresaron que los docentes no se preocupan por conocer a fondo las emociones y necesidades de ellos como padres. De otro lado, para los docentes, las familias delegan todas las responsabilidades a la escuela, sin asumir la tarea de participar en el proceso educativo. En cuanto a la equidad, se encontró que, al no haber una comunicación adecuada para lograr acuerdos entre las partes, los padres perciben que las decisiones son tomadas de forma unilateral por los maestros.

Sobre la competencia profesional, los docentes sostienen que una barrera es la falta de preparación para asumir la tarea de la inclusión sin el apoyo de personal especializado. Los maestros, sin embargo, afirmaron que esto sucede porque el docente muchas veces recibe al estudiante en condición de discapacidad sin haber recibido la formación necesaria; por esto, preferirían que estos estudiantes se fueran a otros centros con niños que poseen sus mismas dificultades. Esto reafirma la afirmación de José María
Fernández Batanero en su estudio sobre las competencias docentes en educación inclusiva (2013): "Todavía son muchos los docentes de educación secundaria que no se encuentran suficientemente cualificados para afrontar la profunda transformación que requiere el sistema educativo bajo el reto que supone dicho modelo" (p. 85). Es bueno resaltar que esto sucede también en otras latitudes; esto puede indicar que llevar a la práctica este proceso de educación inclusiva representa un reto para la formación de los docentes y del mismo sistema educativo (Poon-McBrayer \& Wong, 2013; Young, McNamara \& Coughlan, 2017). Por ejemplo, en Japón un estudio halló que los maestros tenían algunas preocupaciones sobre la implementación de la educación inclusiva para estudiantes con distintas necesidades o barreras, y una baja percepción sobre su autoeficacia (Yada \& Savolainen, 2017). Por esto, se ve que los docentes necesitan mayor competencia profesional y adquirir habilidades y conocimientos para afrontar exitosamente la inclusión, como lo plantean Ashwini Tiwari, Ajay Das y Manisha Sharma (2015); por esto, la capacitación recibida por el cuerpo docente debe ser permanente y darse de acuerdo a las necesidades de la comunidad educativa, no solo delegar esta tarea en los profesionales especializados de apoyo (Sandoval-Mena, Simón-Rueda \& EcheitaSarrionandia, 2012).

Ante este panorama, las propuestas de los participantes apuntaron a la construcción de una interacción cooperativa para incentivar la participación activa de la familia, lo que corrobora lo planteado en estudios previos sobre estrategias para el éxito en la inclusión educativa (Bolívar, 2006; Fontana, Alvarado, Angulo, Marín \& Quirós, 2009; Garnique-Castro \& GutiérrezVidrio, 2012). No obstante, al contrastar estas intenciones con las barreras percibidas, se puede afirmar que no es suficiente generar unas políticas de inclusión, o establecer unos procedimientos pedagógicos para avanzar satisfactoriamente en la intención de lograr la cooperación; para ello, para la implementación de un proceso educativo inclusivo, se requeriría de un cambio metodológico, focalizado en la definición previa de espacios, tiempos, objetivos y reglas, para la creación de pautas funcionales, lo que aumentaría la posibilidad del trabajo conjunto y el aprovechamiento de los recursos que puedan aportarse mutuamente. En otras palabras, se requiere crear un sistema con sus propias reglas y acuerdos básicos, que posibilite encuentros conversacionales efectivos, en cuyo marco se puedan construir nuevos significados y plantear acciones conjuntas entre los diferentes participantes de la comunidad educativa, para optimizar los procesos educativos y superar barreras. Siguiendo este propósito, desde el punto de vista pragmático, el presente estudio aportó una caracterización de los componentes de una 
relación cooperativa, en aras de poder orientar el proceso conversacional hacia identificar y optimizar los recursos y déficits, desde el trabajo en las categorías desarrolladas, es decir, las habilidades sociales comunicativas, los aspectos actitudinales internos y externos y el cumplimiento de roles.

De esta manera, en los grupos, se pudo apreciar que el espacio conversacional tuvo como resultado develar las interpretaciones dominantes, para posteriormente cuestionar premisas y supuestos previos; así se dio paso a una narrativa más benévola y constructiva. Así, la experiencia se puede ver desde el modelo sistémico ecológico, pues en el caso de este grupo de participantes se pasó progresivamente de una problemática individualizada a una construcción de narrativas con sentido colectivo. Como sostiene Nora Aneth Pava-Ripoll (2015):

Las narrativas conversacionales implican procesos interaccionales que permiten escuchar múltiples versiones sobre la realidad que se investiga, comprender la experiencia de estas personas y por consiguiente, se constituyen en una excelente estrategia para orientar el trabajo investigativo con familias y profesores de niños y niñas en condición de discapacidad (p. 217).

Como limitaciones del estudio, las conclusiones son pertinentes específicamente al grupo cultural de referencia, como es característico en los estudios cualitativos; por lo tanto, el mayor peso está en el proceso de construcción narrativa; por tanto, a futuro sería recomendable ampliar su alcance a otros grupos o culturas. Igualmente, no se contempló el tipo de discapacidad de los alumnos cuyos padres participaron en el estudio, o los sesgos cognitivos - la claridad conceptual de los participantes al usar términos específicos relacionados con la discapacidad o los procesos educativos; en este aspecto, se buscó el soporte en una revisión teórica, para dar solidez a la categorización, dado que el estudio dio mayor relevancia a las vivencias emocionales relatadas sobre el proceso conversacional. Otra limitante es el tipo de técnicas utilizadas que, al ser grupales en los relatos de la experiencia, pueden facilitar tomar el dato desde los consensos, pero disminuir la expresión de algunas opiniones individuales, que son más evidentes en otro tipo de técnicas.

\section{Agradecimientos}

Agradecemos a la Universidad de La Sabana por su apoyo en asesoría, formación y tiempos para la investigación.

\section{Sobre los autores}

Luz Mélida Montaño-Contreras es licenciada en Educación con énfasis en Educación Especial de la Universidad Pedagógica Nacional de Colombia, Colombia. Magíster en Psicología de la Salud y la Discapacidad de la Universidad de La Sabana, Colombia. Coordinadora y educadora especial de Educación Inclusiva, Alcaldía de Chía, Colombia. Interés en investigar procesos educativos, inclusión social y discapacidad.

Johana Elizabeth Cerón-Bastidas es terapista ocupacional de la Universidad Mariana de Colombia, Colombia. Magíster en Psicología de la Salud y la Discapacidad de la Universidad de La Sabana, Colombia. Actualmente, trabaja en el Jardín Campestre del Norte, Bogotá, Colombia. Tiene interés en investigar sobre inclusión en discapacidad infantil.

Mario Andrés Ernesto Martín-Padilla es psicólogo. Magíster en Psicología Clínica y de familia. Director de la Maestría en Psicología de la Salud y la Discapacidad. Investiga sobre familias, salud y calidad de vida en distintos contextos. Grupo de investigación: Procesos Psicológicos y Contexto Social. Autor de artículos y capítulos de libro. 


\section{Referencias}

Agudelo-Bedoya, M. \& Estrada-Arango, P. (2013). Constructivismo y construccionismo social: algunos puntos comunes y algunas divergencias de estas corrientes teóricas. Prospectiva, 17, 353-378. DOI: https://doi.org/10.25100/prts.v0i17.1156. Disponible en: http://revistaprospectiva.univalle.edu. co/index.php/prospectiva/article/view/1156/1276

Angenscheidt-Bidegain, L. \& Navarrete-Antola, I. (2017). Actitudes de los docentes acerca de la educación inclusiva. Ciencias Psicológicas 2017; 11 (2): 233243. doi: 10.22235/cp.v11i2.1500. Disponible en: https://revistas.ucu.edu.uy/index.php/cienciaspsi cologicas/article/view/1500/1451

Berger, P. L. \& Luckmann, T. (2001). La construcción social de la realidad. Buenos Aires: Editorial Amorrortu.

Blue-Banning, M.; Summers, J.; Frankland, H. C.; Nelson, L. L. \& Beegle, G. (2004). Dimensions of Family and Professional Partnerships: Constructive Guidelines for Collaboration. Exceptional Children, 70 (2), 167-184. Disponible en: https://kuscholarworks. ku.edu/bitstream/handle/1808/6264/P13_Dimen sionsoffamily9_07.pdf? sequence $=1$ \&isAllowed $=y$

Bolívar, A. (2006). Familia y escuela dos mundos llamados a trabajar en común. Revista de Educación, 339, 119-146. Disponible en: http://www.revista educacion.mec.es/re339/re339a08.pdf

Bronfenbrenner, U. (2002). La ecología del desarrollo humano. Barcelona: Paidós.

Caillé, P. (1992). Uno más uno son tres: la pareja revelada a sí misma. Barcelona: Paidós.

Calderón, M. (2012). La educación inclusiva es nuestra tarea. Educación, 21 (40), 43-58. Disponible en: http://revistas.pucp.edu.pe/index.php/educa cion/article/viewFile/2505/2450

Canales, M. \& Peinado, A. (1995). Grupos de discusión. En J. Gutiérrez \& J. Delgado (comps.). Métodos y técnicas cualitativas de investigación en Ciencias Sociales, 287-316. Madrid: Síntesis.

Cevallos-Mieles, O. (2016). Las habilidades comunicativas, una necesidad formativa del profesional del siglo XXI. Yachana Revista Científica, 5 (1), 130137. Disponible en: http://revistas.ulvr.edu.ec/ index.php/yachana/article/view/290/193

Corbetta, P. (2007). Metodologías y técnicas de la investigación social. Madrid: McGraw-Hill.

Damm-Muñoz, X. (2010). Representaciones y actitudes del profesorado frente a la integración de niños/ as con necesidades educativas especiales al aula común. Revista Latinoamericana de Educación Inclusiva, 3 (1), 25-35. Disponible en: http://sid. usal.es/idocs/F8/ART11920/representaciones_y_ actitudes.pdf
Denzin, N. \& Lincoln, Y. (2000). Handbook of Qualitative Research Lines of Narrative. London: SAGE Publications.

Dimitrova-Radojichich, D. \& Chichevska-Jovanova, N. (2014). Parent's Attitude: Inclusive Education of Children with Disability. International Journal of Cognitive Research in Science, Engineering and Education, 2 (1), 13-17. Disponible en: https:// www.researchgate.net/publication/269336491 Parents_attitude_Inclusive_education_of_chil dren_with_disability

Duk, C. \& Murillo, J. (2013). La colaboración como elemento definitorio de las escuelas inclusivas. Revista Latinoamericana de Educación Inclusiva, 6 (2), 11-13. Disponible en: http://www.rinace.net/ rlei/numeros/vol6-num2/editorial.pdf

Epston, D.; White, M. \& Murray, K. (1996). Una propuesta para re-escribir la terapia. En S. McNamee \& K. Gergen (comps.). La terapia como construcción social, 121-141. Barcelona: Paidós.

Fernández-Batanero, J. (2013). Competencias docentes y educación inclusiva. Revista Electrónica de Investigación Educativa, 15 (2), 82-99. Disponible en: http://redie.uabc.mx/vol15no2/contenidofdzbatanero.html

Fontana, A.; Alvarado, A.; Angulo, M.; Marín, E. \& Quirós, D. (2009). El apoyo familiar en el proceso de integración educativa de estudiantes con necesidades educativas en condición de discapacidad. Educare, 13 (2), 17-35. Disponible en: http:// www.redalyc.org/html/1941/194114401003/ index.html

Freire, P. (1998). ¿Extensión o comunicación? La concientización en el medio rural. Ciudad de México: Siglo Veintiuno Editores.

García-Carmona, M. (2012). Trabajando de forma cooperativa en la escuela: abriendo la puerta a las familias. Revista Electrónica de Investigación Docencia Creativa, 1, 59-66. Disponible en: http:ww w.ugr.es/local/miguelgr/ReiDoCrea-Vol.1-Art.8Garcia.pdf

Garnique-Castro, F. \& Gutiérrez-Vidrio, S. (2012). Educación básica e inclusión: un estudio de representaciones sociales. Magis, Revista Internacional de Investigación en Educación, 4 (9), 577-593. Disponible en: http://revistas.javeriana.edu.co/ index.php/MAGIS/article/view/3577

Garreta i Bochaca, J. (2007). La participación de los progenitores en las instituciones escolares: realidades e imágenes. Lleida: Universitat de Lleida.

Gergen, K. (1996). Realidades y relaciones: aproximaciones a la construcción social. Barcelona: Ediciones Paidós Ibérica.

González-Rojas, Y. \& Triana-Fierro, D. (2018). Actitudes de los docentes frente la inclusión de estudiantes 
con necesidades educativas especiales. Educación y Educadores, 21 (2), 200-218. doi: 10.5294/edu.2018.21.2.2. Disponible en: http:// educacionyeducadores.unisabana.edu.co/index.php/eye/article/ viewFile/8783/4866

Hildebrand, D. von (2004). La importancia del respeto en la educación. Revista Científica de América Latina, el Caribe, España y Portugal, 7, 221 228. Disponible en: http://www.redalyc.org/articulo.oa?id=83400715

Jiménez-León, I. (2008). La relación familia y escuela. Jaén, Andalucía: Íttakus. Disponible en: http://caps.educacion.navarra.es/primaria2/ files/La\%20relaci\%C3\%B3n\%20familia\%20escuela.pdf

Martínez-Navarro, E. (2010). Ética profesional de los profesores. Madrid: Universidades Jesuitas, UNIJES.

Montoya-Arango, V.; García-Sánchez, A. \& Ospina-Mesa, C. (2013). Andar dibujando y dibujar andando: cartografía social y producción colectiva de conocimientos. Nómadas, 40, 191-205. Disponible en: https:// www.redalyc.org/articulo.oa?id $=105131005013$

Morey, L. A. (2009). Cuestión de confianza. Vuelos, 8 (8), 30-40. Disponible en: https://revistas.upc.edu.pe/index.php/pozo/article/view/235/190

Okuda, M. \& Gómez-Restrepo, C. (2005). Métodos en investigación cualitativa: triangulación. Revista Colombiana de Psiquiatría, 18 (1), 118-124. Disponible en: http://www.redalyc.org/articulo.oa?id=80628403009

Pava-Ripoll, N. A. (2015). Narrativas conversacionales con familias y docentes de niños y niñas en condición de discapacidad: un aporte metodológico. Interdisciplinaria, 32 (2), 203-222. Disponible en: http:// www.redalyc.org/articulo.oa?id=18043528001

Peñafiel-Martínez, F. (2012). Planteamiento inclusivo de la participación de la familia en contextos de intervención con alumnos en condición de discapacidad. Revista de Educación Inclusiva, 5 (2), 117-127. Disponible en: http://www.revistaeducacioninclusiva.es/index.php/REl/ article/view/237/231

Poon-McBrayer, K. \& Wong, P. (2013). Inclusive Education Services for Children and Youth with Disabilities: Values, Roles and Challenges of School Leaders. Children and Youth Services Review, 35 (9), 15201525. http://dx.doi.org/10.1016/j.childyouth.2013.06.009

Sales-Ciges, M.; Ferrández-Berrueco, R. \& Molinet-García, O. (2012). Escuela intercultural inclusiva: estudio de caso sobre procesos de autoevaluación. Revista de Educación, 358, 153-173. DOI: 104438/1988-592X-RE-2012-358-187. Disponible en: https://www. mecd.gob.es/dctm/revista-de-educacion/articulos-re358/re35808. pdf?documentld=0901e72b8128205d

Sandoval-Mena, M.; Simón-Rueda, G. \& Echeita-Sarrionandia, G. (2012). Análisis y valoración crítica de las funciones del profesorado de apoyo desde la educación inclusiva. Revista de Educación, 117-137. DOI: 10.4438/1988-592X-RE-2012-EXT-209. Disponible en: https://www. mecd.gob.es/dctm/revista-de-educacion/articulosre2012/re201205. pdf?documentld=0901e72b81426f62

Strauss, A. \& Corbin, J. (2002). Bases de la investigación cualitativa. Técnicas y procedimientos para desarrollar la teoría fundamentada. Medellín: Universidad de Antioquia.

Sukys, S.; Dumciene, A. \& Lapeniene, D. (2015). Parental Involvement in Inclusive Education of Children with Special Educational Needs. Social Behavior and Personality, 43 (2), 327-338. DOI: https://doi. org/10.2224/sbp.2015.43.2.327. Disponible en: https://www.sbpjournal.com/index.php/sbp/article/view/4334 
Terrón, E.; Alfonso, C. \& Díez, E. (2003). Construir la comunicación entre la familia y la escuela como una relación de confianza. Barcelona: Graó Editorial.

Tiwari, A.; Das, A. \& Sharma, M. (2015). Inclusive Education A "Rhetoric" or "Reality"? Teachers' Perspectives and Beliefs. Teaching and Teacher Education, 52, 128-136. http://dx.doi.org/10.1016/j.tate.2015.09.002. Disponible en: https://www.academia.edu/16964440/Inclusive education a rhetoric or reality Teachers perspectives and beliefs

Trevithick, P. (2006). Habilidades de comunicación en intervención social: manual práctico. Madrid: Narcea.

Turnbull, A. \& Turnbull, H. R. (1990). Families, Professionals, and Exceptionality: A Special Partnership. Columbus, Ohio: Merrill Publication Company.

Turnbull, A.; Turnbull H. R. \& Kyzar, K. (2009). Cooperación entre familias y profesionales como fuerza catalizadora para una óptima inclusión: enfoque de los Estados Unidos de América. Revista de Educación, 349, 69-99. Disponible en: http://www.ince.mec.es/revistaeducacion/ re349/re349 04.pdf

Valdés, Á. A. \& Sánchez-Escobedo, P. A. (2016). Las creencias de los docentes acerca de la participación familiar en la educación. Revista Electrónica de Investigación Educativa, 18 (2), 105-115. Disponible en: https://redie.uabc.mx/redie/article/view/1174/1446

Valles, M. S. (1999). Técnicas cualitativas de investigación social. Madrid: Síntesis.

Warren, J.; Vialle, W. \& Dixon, R. (2016). Transition of Children with Disabilities into Early Childhood Education and Care Centres. Australasian Journal of Early Childhood, 41 (2), 18-27. Disponible en: https://scho lars.uow.edu.au/display/publication114586

White, M. \& Epston, D. (1993). Medios narrativos para fines terapéuticos. Barcelona: Paidós.

Widding, G. \& Berge, B. M. (2014). Teachers' and Parents' Experiences of Using Parents as Resources in Swedish Primary Education. Procedia - Social and Behavioral Sciences, 116 (21), 1587-1593. http://dx.doi. org/10.1016/j.sbspro.2014.01.439

Yada, A. \& Savolainen, H. (2017). Japanese In-Service Teachers' Attitudes toward Inclusive Education and Self-Efficacy for Inclusive Practices. Teaching and Teacher Education, 64, 222-229. http://dx.doi. org/10.1016/j.tate.2017.02.005

Young, K.; McNamara, P. M. \& Coughlan, B. (2017). Authentic InclusionUtopian Thinking? - Irish Post-Primary Teachers' Perspectives of Inclusive Education. Teaching and Teacher Education, 68, 1-11. http:// dx.doi.org/10.1016/j.tate.2017.07.017 